\title{
CORRELATION OF LOWER LIMB STRENGTH, POWER, WAIST-HIP RATIO AND BMI WITH A SITTING-RISING TEST IN 18-35 YEARS AGE GROUP
}

\author{
Neha Gotmare, Simran Narang, Ruchi Chandra, Maneesha Deshpande \\ VSPM College of Physiotherapy, Nagpur, Maharashtra, India.
}

\begin{abstract}
Purpose: To investigate the co-relation of sitting-rising test scores with measures of lower extremity strength, power, and body compositions (body mass index and waist-hip ratio). Furthermore, to find out the level of physical activity in the targeted population. Methods: Participants aged between 18-35 years $(n=131)$ of both genders were recruited in this study. Along with performing sitting-rising test, anthropometric and demographic data were collected to calculate body mass index and waist-hip ratio. Lower extremity strength was assessed using a 30 -second chair stand test, and power was assessed using a triple hop test for distance. Also, the Rapid Assessment Disuse Index questionnaire was given to dividing the population into two groups depending on whether they are involved more in physical activity or not. Results: Sitting-rising test scores showed a positive correlation with lower extremity strength and power, negative correlation with body mass index, and no co-relation with waist-hip ratio. Further, 76 participants out of 131 were less physical activity and had more sedentary behavior. Conclusion: Sitting-rising from the floor in young adults is influenced by the strength and power of lower extremities and body mass index except for the waist-hip ratio.
\end{abstract}

Keywords: Sitting-rising test; Lower extremity strength and power; RADI score.

\section{INTRODUCTION}

Sitting and rising from the floor is the primary functional task required for independence [1]. In ancient times, many activities (like cooking, washing clothes and utensils, sweeping, etc.) were done by sitting down whereas now modern technology has lessened the physical demands and many activities are done with just one press of a button. Inability to perform these actions is closely related to the risk of falling. To have a successful transition from standing to sitting or rising from the floor, proper levels of muscle strength, muscle power, flexibility, coordination, body composition, and balance are required [1].

Nowadays, most people teach themselves an environment that not only limits the physical activity but requires prolonged sitting (at work, home, and in communities). Work sites, schools, homes, and public spaces, etc. have been re-engineered in a way that minimizes human movement and physical activity. According to WHO, adults (18-64 year) should do at least 150 minutes of moderate intensity physical activity throughout the week or at least 75 minutes of vigorous intensity physical activity or an equivalent combination [2]. Adequate physical activity and fitness are considered as one of the key factors in current public health promotion.

Physical fitness reflects the effects of regular physical activity and hence is an important prognostic factor of health [3]. Physical fitness is a multidimensional

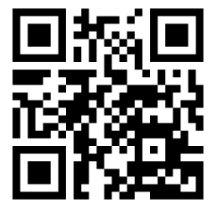

DOI: $10.31878 /$ ijcbr.2019.61.05

eISSN: $2395-0471$

pISSN: 2521-0394 concept that has been defined as a set of attributes that people achieve that relates to the ability to perform physical activity. It comprises of skill related, healthrelated, and physiologic component [4]

Skill-related components of physical fitness include agility, balance, coordination, speed, power, and reaction time. Health-related components include cardiovascular endurance, muscular strength and endurance, flexibility, and body composition.

Physiologic fitness includes metabolic fitness, morphological fitness, bone integrity, etc.

In World Health Survey prevalence of physical inactivity in India was $9.3 \%$ in males and $15.2 \%$ in females. A study was done by Shah et al. in 2005 in 6 regions on India (Delhi and Ballagarh in north, Chennai, and Trivandrum in the south, Nagpur in west and Dibrugarh in the east) showed overall inactivity level was $12.6 \%$ in males and $18.9 \%$ in females. Recently, Anjana et al. in 2014 showed that total of 392 million individuals are inactive in India [5].

Sedentary behaviour is a cluster of behaviour adopted in sitting or lying posture where little energy is being spent [6]. Increased sitting time is associated with increased cardiovascular risk, all-cause mortality, and a variety of physiological and psychological problems independent of confounding factors in adults [7].

Claudio Gil Araujo, of Gama Filho University in Rio de Janeiro, Brazil, proposed a simple method to assess the ability to sit and rise from floor termed "sitting-rising test (SRT)." Sitting and rising test objectively quantifies the number of supports needed and the presence or absence of balance stability for these actions [1]. SRT mainly examines the quality of movement [8]. SRT assess the components of musculoskeletal fitness

Correspondence: Dr. Neha Gotmare, Associate Professor, VSPMs College of Physiotherapy, Nagpur, MHS. Email: dr.nehagotmare24@gmail.com

(C) Authors; 2020. International Journal of Clinical and Biomedical Research, Sumathi Publications.

This is an Open Access article which permits unrestricted non-commercial use, provided the original work is properly cited.

(CC BY-NC-SA 4.0) 
through evaluation of the subject's ability to sit and rise from the floor. When standing up, ankle plantar flexors work concentrically while dorsiflexors contract eccentrically. Excellent ankle strength is required to stand from sitting on the floor without using the hands and without swaying or losing balance [8]. In comparison with other physical fitness tests like Get-up and Go test, SRT does not require any equipment, neither it requires a walkway, nor it is depended on time. It is even safe and can be done in a short period of time and can be reliably scored [1]. SRT scores have been shown to have high reliability in assessing healthy subject [8]. The ability to achieve a high SRT score could reflect the capacity to successfully perform activities of daily living. Moreover high SRT score is related to low chances of fall and low scores in SRT are reflects the degree of impairment in the musculoskeletal fitness [1]. A study done in Brazil says that excess body weight impairs sitting and rising actions in adults [9]. According to the previous study, SRT score in the range of 8-10 indicates a low risk of death, whereas low score is associated with $>6$ fold higher all-cause mortality in men and women. Young adults (18-35 years) are selected in this study as the muscle strength starts getting impaired after 35 years of age due to a decrease in muscle mass, reduction in the size and number of type II fibres, as well as reduced number of motor unit and some of the chronic hypokinetic diseases such as heart attack, diabetes, stroke, cancer, etc. that are associated with lack of physical activity, are relatively less prevalent in young adults $[1,10]$. Also, the prevalence of musculoskeletal disorder in India is low in the age group of 18-30 years. Moreover, muscle power declines more rapidly than muscle strength with aging, which is associated with a decrease in functional ability [10].

Lower extremity muscle integrity maintenance is important to delay and prevent the onset of disability, physical weakness, or dependency in the later stage of life. Therefore, it becomes important to assess the lower limb strength and power of young adult at an early age.

"Chair stand test" or "sit to stand" test is used as a proxy indicator to measure lower extremity strength because of its feasibility to administer when compared to the isokinetic knee extensor strength which is the most acceptable method [11]. Advantage of using this test is that it does not require any special equipment nor any specialized training or trained person [12]. A study conducted by Jones et al. reported that counting the number of times a client stands up for 30 seconds is more reliable than existing test variations which checks the time one takes to stand for a total of 5 or 10 times [13]. These variations differ in only one thing that 5 repetitions sit to stand or 10 repetitions sit to stand measures speed and power whereas 30 -second sit to stand serves as an indicator for strength and endurance. Hence, they are not to be used interchangeably [11]. Furthermore, the study concluded that 30 -second chair stand test provides a reasonably reliable and valid indicator and good test-retest reliability of lower body strength in generally active, community-dwelling older adults. Therefore, this test was taken for the current study [13].

Single leg hop tes twas designed to assess functional performance in an injured extremity. Researchers initially used this test in patients with postsurgical knee patient's specifically anterior cruciate ligament reconstructions and determined hop test's validity as an objective clinical measure. Single leg hop test and vertical jump test are both considered functional tests and are reported in the literature to be useful as part of a battery of tests to determine readiness to participate in the activity. The advantage of a triple hop test over the standard vertical jump is that each leg can be evaluated independently, so asymmetries or weakness may be identified. Also, this test requires minimal space, time, and equipment and may be especially helpful in places where resources are limited, and sophisticated testing equipment is not available. This test is a valid predictor of lower limb power that can provide similar results to that of vertical jump, was included in the current study as an assessment test for lower extremity power [14].

Sitting-rising test is influenced by excess body weight, especially in women, due to a different pattern of body composition [9]. Therefore, we hypothesized that body composition might be related to the sitting-rising test and hence, body mass index (BMI) and waist-hip ratio (WHR) was calculated and correlated.

As there is less relevant data between musculoskeletal fitness with a health outcome in young adults than in elderly, the present study was carried out to observe which of the components of musculoskeletal fitness, i.e. muscle strength and power of lower limb, BMI, and WHR affects the sitting-rising test.

Aim: To correlate lower limb strength, lower limb power, body mass index (BMI) and waist-hip ratio (WHR) on the sitting-rising test in the age group 18-35 years".

\section{MATERIAL AND METHODOLOGY}

Study design: Co-relational Study.

Ethical approval: Prior to the test, the purpose and procedures of the study were explained to all the eligible subjects and all gave their written consent to participate in the study.

Study setting: V.S.P.M's College Of Physiotherapy and other

colleges in Nagpur were purposefully selected considering feasibility and convenience.

Target population: $18-35$ years

Duration of study: 1 year and 6 months was utilised for the completion of the study.

Sample size: Sample size was determined by prevalence of musculoskeletal disorder [16] as the main outcome and with following assumptions: Anticipated prevalence of musculoskeletal disorder in adult population $=9.3 \%$, Confidence interval $=95 \%(\alpha$ error $=5 \%)$, Relative precision $=10 \%$, Required sample size: 131

\section{METHOD}

Inclusion criteria: The inclusion criteria included both genders and apparently healthy individuals

Exclusion criteria: whereas subjects any neurological impairments, recent abdominal or lower limb surgeries, 
pregnant women, any lower extremity trauma like a fracture, any ankle conditions, with any pain and participants not willing to participate were excluded.

Anthropometric and demographic was collected. Apart from the sitting-rising test (SRT), the subjects were given a Rapid Assessment Disuse Index (RADI) questionnaire, and were also required to complete the 30 second chair stand test for lower limb strength; triple hop test for lower limb power and collected anthropometric and demographic data was used to calculate BMI and waist-hip ratio (WHR). The testing order was randomised, and 2-minute rests were given between each test to reduce fatigue.

The SRT evaluates musculoskeletal fitness. It was administered on a non-slippery surface, with the subject standing barefoot and wearing clothes that will not restrict the movement. Before initiation of the test subject were instructed as "Without worrying about the speed of movement, try to sit and then rise from the floor, using the minimum support that you believe is needed." The score begins with a maximum of 5 points separately for sitting and rising. One point will be subtracted for each support, that is, hand, forearm, knee, or side of the leg and an additional 0.5 point was subtracted if there is partial loss of balance during the movement. The crossing of legs for either sitting or rising from the floor was allowed, while the sides of the subject's feet will not be used for support. This test was conducted only once [1].

Lower limb strength was measured using a 30-second chair stand test where subjects were asked to sit on a standard chair (approximate. $43.20 \mathrm{~cm}$ ) without armrest. They were instructed to settle themselves half of the buttock with straighten back and crossing wrists in front of the chest and were then asked to stand up and sit down repeatedly for 30 seconds and numbers of stands were counted. The test was conducted trice, and best of three were used for results $[11,12]$.

Triple hop test was used to assess lower limb power. Subjects were asked stand on the designated testing leg with a great toe on the starting point. They performed 3 consecutive maximal hops forward on the same limb. Arm swings are allowed. The distance was measured from the starting point to the point where the heel will struck the ground after completion of the third hop. The procedure was performed with the dominant leg [14]. Three test trials were given, and the best of the three distances was used for results. The maximum distance achieved was recorded in centimeters $(\mathrm{cm})$

Body mass index (BMI) was calculated by standard formula "Weight $(\mathrm{kg}) /$ Height $\left(\mathrm{m}^{2}\right)$ " whereas the waisthip ratio was calculated by "Waist measurement (inches) / Hip measurement (inches)."

Rapid Assessment Disuse Index (RADI) is a brief assessment tool designed to quickly identify subjects with a high level of sitting and low daily physical activity [17]. The participants were asked to complete the matrix with reference to the past week, month, and year. It comprises of 3 questions aimed at measuring sitting time as well as general moving and stair climbing behaviour. The two RADI questions related to lifestyle activity (moving about and stair climbing) are reverse scored, that is, higher scores are indicative of less moving about, and fewer stairs climbed; whereas the sitting score is directly scored, that is, a higher score is indicative of more sitting. RADI score for each column is between 3 to 15 , and cumulative score $(a+b+c)$ between 9 to 45 . A score of 26 or more indicates increase sitting and reduced physical activity (i.e., disuse) [17].

Statistical analysis: Data was coated and analyzed in statistical STATA, version 10.1, Inferential statistic includes estimation of the prevalence of musculoskeletal disorder express as a percentage with $95 \%$ confidence interval. The correlation between 4 influencing factors (lower limb strength, lower limb power, BMI, and WHR) with SRT was assessed by calculating Pearson's Correlation Coefficient (r). Significance of which was tested by t-test. $\mathrm{P}<0.05$ will be considered statistically significant.

\section{RESULTS}

The given table 1 shows the correlation between sittingrising test scores and the outcome measures. Since all variables were normally distributed, Pearson correlation coefficients were used.

Table 1. Correlations between SRT scores and the outcome measures $(n=131)$

\begin{tabular}{ll}
\hline Outcome measures & $\begin{array}{l}\text { Sitting-Rising test } \\
\text { (Pearson's r) }\end{array}$ \\
\hline 30-second chair stand test & 0.3227 \\
Triple hop test & 0.1839 \\
Body mass index (BMI) & -0.5515 \\
Waist-hip ratio (WHR) & -0.05272 \\
RADI score & \\
More than 26 & 0.1661 \\
Less than 26 & -0.1853 \\
\hline
\end{tabular}

Sitting-rising score was positively correlated with lower limb strength and power and in participants with more than 26 RADI score, whereas negatively correlated with BMI and in participants with less than 26 RADI score. However, there was a nocorrelation between sittingrising test scores and waist-hip ratio.

The given figure 1-4 shows the correlation of sittingrising test with 30-second chair stand test, triple hop test, BMI, and WHR. Figure 5 shows the division of participants in two groups according to RADI score, that is, more than 26 and less than 26. Correlation of these two groups with the sitting-rising test score is shown in figure 6 and figure 7.

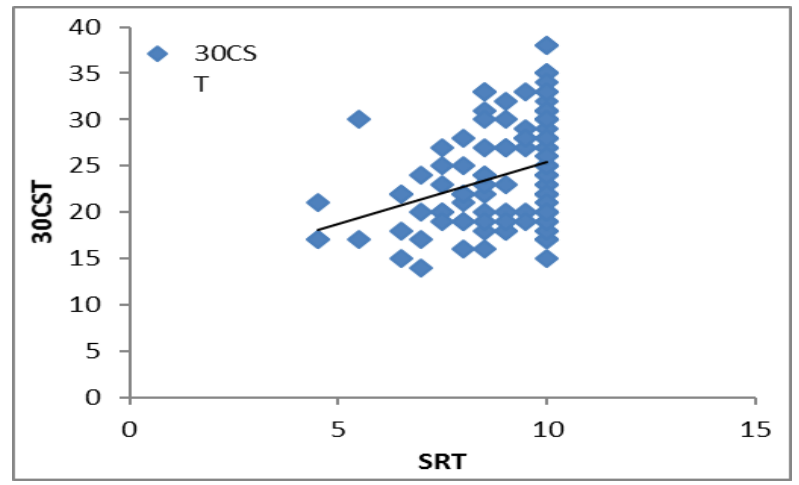

Fig 1: Correlation of sitting-rising test with 30 -second chair stand test 


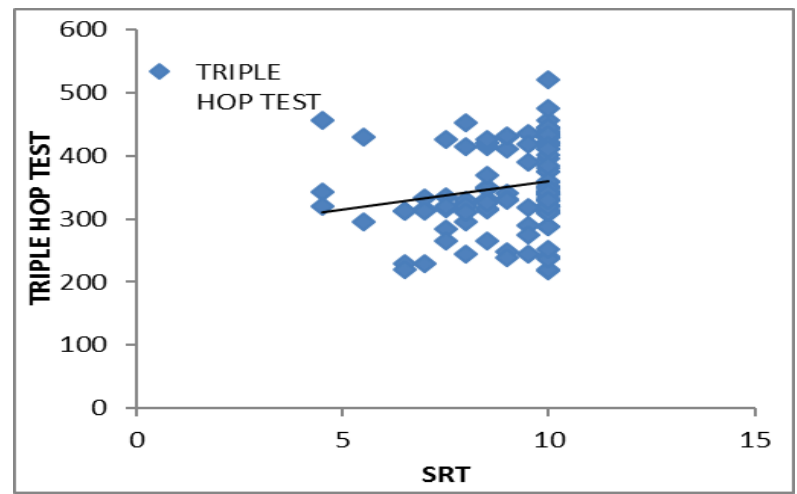

Fig 2: Correlation of sitting rising test with triple hop test

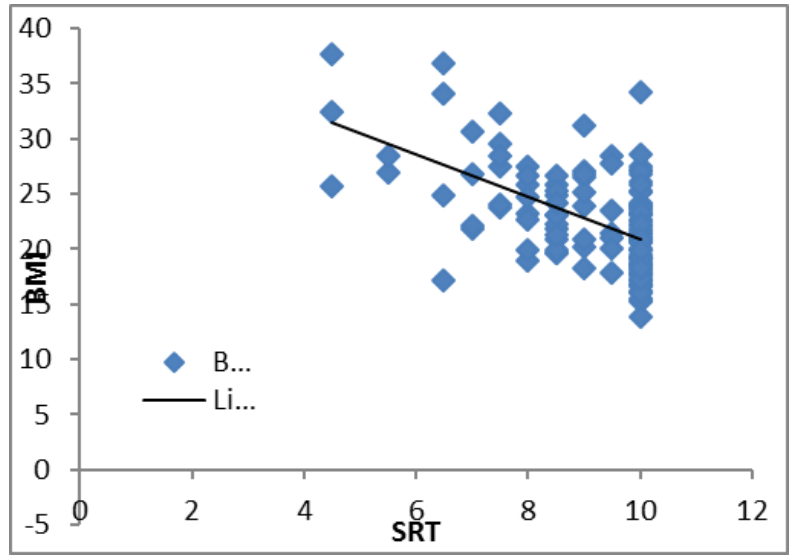

Fig 3: Correlation of sitting rising test with body mass index

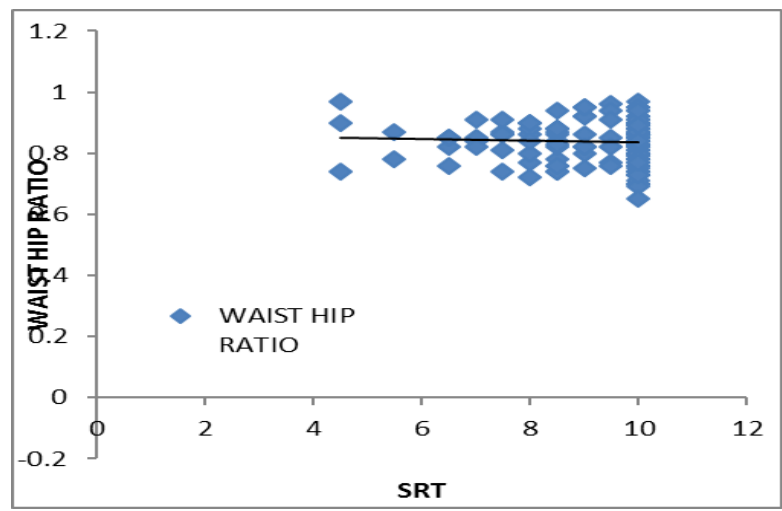

Fig 4: Correlation of sitting rising test with waist-hip ratio

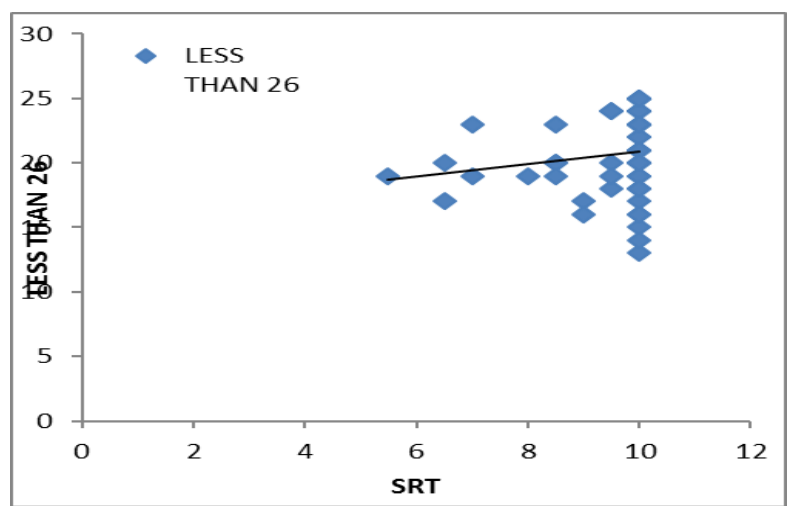

Fig 6: Correlation of sitting rising test with subjects less than 26 score

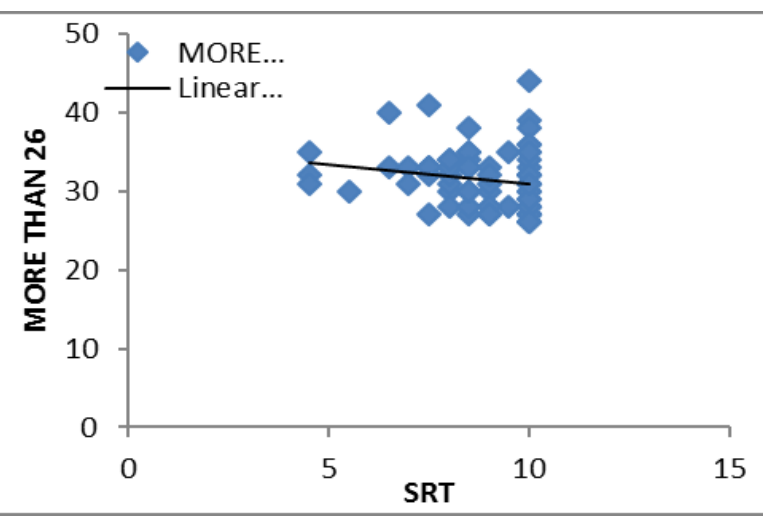

Fig 7: Correlation of sitting rising test with subjects more than 26 score

RADI score: This graph depicts that 55 subjects out of 131 have spent less time in sitting and are more physically active and 76 subjects are more into sitting and less physically active.

\section{DISCUSSION}

The purpose of the study was to correlate the sittingrising test with some of the physical fitness components, namely strength, power, BMI, and waist-hip ratio in healthy young adults. Rising from the floor is the essential activity of daily living requires an adequate amount of muscle power, balance, flexibility, and strength [1]. Brito et al. (2013) concluded that high levels of flexibility could provide the ability to sit and rise from the floor properly [15]. In the present study, the results showed a positive correlation of sitting-rising test (SRT) with lower extremity strength and power but a negative correlation with BMI and no correlation with waist-hip ratio (WHR). The following discussion addresses the relationship noted between SRT and each of the dependent measures.

Lower extremity strength was assessed using a "30second chair stand test," which is a valid indicator of strength. However, to my knowledge, no other study till date has correlated SRT with lower limb strength. Standing up from cross-leg sitting requires combined interaction of associated body segments that includes flexion of trunk and hip to bring the center of mass forward, that is followed by extension of both lower limbs and lastly the trunk extension to raise body mass in the vertical direction over the feet [15]. On the other hand, rising from the chair has four phases, as described by M.Schenkman et al. (1990) [18]. Phase 1 is the flexion momentum phase that includes anterior flexion of trunk and pelvis so as to move the center of mass anteriorly and upwardly and this phase requires sufficient amount of hip flexor and ankle dorsiflexor strength. Phase 2 is the momentum transfer phase that starts when buttocks are entirely off the seat and ends when maximal ankle dorsiflexion is achieved. This phase requires adequate knee extensor strength. Phase 3 is the extension phase in which there is a complete extension of the hip, and the major task of this phase is to translate the body vertically. Hence an ample amount of hip and knee extensor strength is required for this phase. Phase 4 is called the stabilization phase that comprises of anterior and posterior sway in standing and thus needs an abundant amount of ankle plantarflexor 
strength. This ankle plantar flexor strength is the most contributing one followed by knee and hip strength, and also it plays an essential role in stabilizing the body at the end of standing in the chair stand test. It is, therefore, reasonable to believe that loss of lower limb strength will affect the successful transition of rising from the floor. Kang Hee Cho et al.(2012) used the same 30-second chair stand test as a lower limb strength indicator and stated that loss of strength is associated with increased risk of fall [12]. Also, the 30-second chair stand test initiated at mid-segment level (from the chair) whereas the sitting-rising test initiates at a low level (from the floor). Therefore there is a moderate correlation

Power of lower extremities was assessed using triple hop test with the dominant leg. This test is previously used in studies conducted on athletes, patients with an anterior cruciate ligament injury and reconstruction [14], and in women with patellofemoral pain [19]. Triple hop has two basic phases viz. propulsion phase and landing phase. During hop test, many forces act on the body which includes gravity, contact with other objects or friction and internal forces that are exerted within the body. Collapse can occur after a hop due to forces that act on a lower limb, which results in accelerated hip and knee flexion and ankle dorsiflexion. In a successful landing, this collapse is avoided by applying counter extensor moments i.e. deceleration forces that work eccentrically to absorb the kinetic energy from the skeletal system and stop the person from falling [20]. A study was conducted by Sofia A. Xergia et al. (2013) on controlled group and anterior cruciate ligament reconstruction (ACLR) group, concluded that subjects in ACLR group had less knee flexion and ankle dorsiflexion after 6 to 9 months following ACLR in both phases of hop test with involved limb as compared to the intact limb. Adequate knee flexion allows greater propulsion force that helps to perform better in the hop test [21]. Triple hop test requires the generation of leg power to maximize the horizontal distance and sitting-rising test requires rising from cross leg sitting to standing at only one place. Therefore, there is a weak correlation.

A study conducted by Andrea L. et al. (2011) concluded that higher BMI levels are associated with poorer mobility but not with standing balancing [22]. Also, higher BMI is associated with poor chair stand performance (Hardy R. et al. 2013) [23]. The result from the current study clearly signifies that on increment in BMI will decrease the SRT scores. Change in weight has great impact on the action on the rising from the floor because of the force of gravity that acts in opposition to the movement, and hence it becomes difficult for the person to lift his body against gravity without support. Similar results were given by Djalma Rabelo Ricardo et al. (2000) in their study when conducted on 461 Brazilian people [15].

It is a well known fact that body fat distribution differs in upper and lower extremities Increased mechanical load, as well as fat distribution, reflected by an increase in waist-hip ratio, have an effect on strength parameters [24]. Greater waist circumference tended to have poorer fitness test performance (Lockie RG et al.) [25]. A study by L Otten et al. (2016) stated that increase in WHR is associated with increased knee extension strength in women but not men whereas there was no correlation of WHR with handgrip strength[24].

RADI scores are a brief assessment tool that combines both sitting and lifestyle activity [17].Cumulative RADI scores are directly correlated with sedentary time and inversely correlated with sedentary breaks and decreased physical activity [17].It assists in recommendations for decreasing sedentary behaviour and increasing physical activity at a point of care and avoid further health-related complication due to increased sitting. In the current study, 55 subjects out of 131 have to spend less time sitting and are more physically active, whereas 76 subjects were more into sitting and less physically active. When these two groups were correlated separately with SRT, we got a weak negative correlation of sitting-rising test with subjects scoring more than 26 in RADI which indicates that subjects with more sitting time have lower sittingrising test scores, whereas, a weak positive correlation of sitting-rising test with subjects scoring less than 26 in RADI indicating that subjects with less sitting time and with more physical activity have higher sitting-rising test scores.

\section{CONCLUSION}

The action of sitting and rising from the floor in young adults is influenced by the strength and power of lower extremities and body mass index except forwaist-hip ratio.

\section{Conflict of interest: Nil}

Source of funding: Nil

\section{REFERENCES}

[1] Brito LB, Ricardo DR, Araújo DS. Ability to sit and rise from the floor as a predictor of all-cause mortality. Eur J Prev Cardiol. 2014;21:892-8.

[2] World Health Organization (WHO) Physical activity 23 February 2018[internet]; Available from : http://www.who.int/en/news-room/factsheets/detail/physical-activity.

[3] Suni JH, Rinne MB, Ruiz JR. Retest Repeatability of Motor and Musculoskeletal Fitness Tests for Public Health Monitoring of Adult Populations. J Nov Physiother. 2014;4:194.

[4] Vivian HH. Advances Fitness Assessment Exercise Prescription ( ${ }^{\text {rd }}$ edition), Chapter 1, Page -2 .

[5] Ranjit MA, Rajendra Pradeepa, Ashok KD, Mohan Deepa, Anil Bhansali. Physical activity and inactivity patterns in India - results from the ICMR-INDIAB study (Phase-1) [ICMR-INDIAB5], Int J Behav Nutr Phys Act. 2014; 11: 26.

[6] Juliet AH, Sebastien FM, Chastin, Dawn AS. Prevalence of Sedentary Behavior in Older Adults: A Systematic Review. Int. J. Environ. Res. Public Health. 2013;10:6645-61

[7] Elin Kolle, Ulf Ekelund. Is Sitting Time a Strong Predictor of Weight Gain?. Curr Obes Rep (2013) 2:77-85. 
[8] Shamay SM Ng, Shirley Sm. FoNg, WayNe S. ChaN, BeN K.y. huNg, et.al. : The sitting and rising test for assessing people with chronic stroke. J. Phys. Ther. Sci. 2016;28:1701-8.

[9] Ricardo DR, Arau' jo CGS. Teste de sentarlevantar: influe^ ncia do excesso de peso corporal em adultos. Rev Bras Med Esporte 2001;7:45-52.

[10] Committee on Fitness Measures and Health Outcomes in Youth; Food and Nutrition Board; Institute of Medicine; Pate R, Oria M, Pillsbury L, editors. Fitness Measures and Health Outcomes in Youth. Washington (DC): National Academies Press (US); 2012;10:6.

[11] Erick KM, Michael AH, Philip H, Joseph M. Wisenbaker : Repeated Chair Stands as a Measure of Lower Limb Strength in Sexagenarian Women, Journal of Gerontology: Medical Sciences America 2004;59(11):1207-12.

[12] Kang Hee Cho, Soo Kyung Bok, Young-Jae Kim, Seon Lyul Hwang. Effect of Lower Limb Strength on Falls and Balance of the Elderly, Ann Rehabil Med. 2012;36(3):386-93.

[13] Jones, Jessie \& Rikli, Roberta CB, Beam William. (1999). A 30-s Chair-Stand Test as a Measure of Lower Body Strength in Community-Residing Older Adults. Research quarterly for exercise and sport. 1999;70:113-9.

[14] Tyler HR, Sandra JS, Randy JS, David HP. TripleHop Distance as a Valid Predictor of Lower Limb Strength and Power. Journal of Athletic Training 2008;43(2):144-51.

[15] Brito LBB, AraújoDSMS, Araújo CGS: Does flexibility influence the ability to sit and rise from the floor? Am J Phys Med Rehabil 2013;92:241-7

[16] Sharma R, editor. Epidemiology of Musculoskeletal Conditions in India. New Delhi, India: Indian Council of Medical Research (ICMR); 2012.

[17] Kerem Shuval, Harold W Kohl III, Ira Bernstein, Dun Iei Cheng, Kelley Pettee Gabriel, Carolyn E Bariow, Liu Yinghui and Loretta DiPietro : Sedentary behaviour and physical inactivity assessment in primary care: the Rapid Assessment Disuse Index (RADI) study. Br J Sports Med. 2014;48(3): 250-5.

[18] Margaret Schenkman, Rlchard AB, Patrick 0R, Robert WM, Andrew WH. Whole-Body Movements During Rising to Standing from Sitting. Physical TherapyNolume. 1990;70:51-61.

[19] Bley AS, Correa JCF, Reis ACD, Rabelo NDDA, Marchetti PH, et al. (2014) Propulsion Phase of the Single Leg Triple Hop Test in Women with Patellofemoral Pain Syndrome: A Biomechanical Study. PLoS ONE 9(5): e97606. doi:10.1371/ journal.pone.0097606.

[20] Storevold, Annika. Knee kinematic and kinetic characteristics of landing after hop : ACL injured subjects before and after rehabilitation,
Masteroppgave - Norges idrettshøgskole, 2009

[21] Sofia AX, Evangelos Pappas, Franceska Zampeli, Spyros Georgiou, Anastasios DG. Asymmetries in Functional Hop Tests, Lower Extremity Kinematics, and Isokinetic Strength Persist 6 to 9 Months Following Anterior Cruciate Ligament Reconstruction, Journal of Orthopaedic \& Sports Physical Therapy. 2013; 43(3):154-62.

[22] Andrea LH, David MW, Elizabeth SH, Stephanie AS, Jennifer SB. Association of Body Mass Index With Self-Report and Performance-Based Measures of Balance and Mobility; PHYS THER. 2011; 91:1223-34.

[23] Hardy R, Cooper R, Aihie SA, Ben-Shlomo Y, Cooper C. Body Mass Index, Muscle Strength and Physical Performance in Older Adults from Eight Cohort Studies: The HALCyon Programme. PLoS ONE. 2013;8(2): e56483.

[24] Otten L, Bosy-Westphal, Ordemann J, Rothkegel E, Stobäus N, Elbelt U, Norman K. Abdominal fat distribution differently affects muscle strength of the upper and lower extremities in women, European Journal of Clinical Nutrition. 2016;1-5.

[25] Lockie RG, Ruvalcaba TR, Stierli M, Dulla JM, Dawes JJ, Orr RM. Waist Circumference and Waist-to-Hip Ratio in Law Enforcement Agency Recruits: Relationship to Performance in Physical Fitness Test, PMID:30204656, DOI:10.1519/JSC.0000000000002825.

Annexure (PTO) 


\begin{tabular}{|c|c|c|c|}
\hline Rapid Assessment Disuse Index & $\begin{array}{l}\text { In the past } \\
\text { WEEK }\end{array}$ & $\begin{array}{l}\text { In the past } \\
\text { MONTH }\end{array}$ & $\begin{array}{l}\text { In the past } \\
\text { YEAR }\end{array}$ \\
\hline \multicolumn{4}{|l|}{$\begin{array}{l}\text { About how many hours a day do you typically spend MOVING AROUND } \\
\text { ON YOUR FEET? } \\
\text { Include moving around on your feet during work related activities, household work (like } \\
\text { cleaning). yard work (like mowing the lawn/ raking), walking (from place to place or for } \\
\text { leisure/ sports), and running at a slow or fast pace. } \\
5=\text { less than } 1 \text { hour/day } \\
4=1 \text { to less than } 3 \text { hours/day } \\
3=3 \text { to less than } 5 \text { hours/day } \\
2=5 \text { to less than } 7 \text { hours/day } \\
1=7 \text { or more hours/day }\end{array}$} \\
\hline \multicolumn{4}{|l|}{ 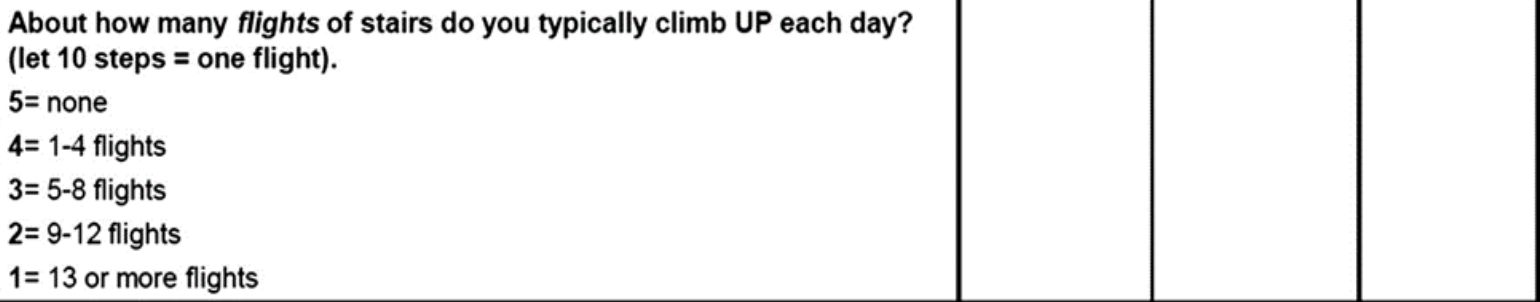 } \\
\hline 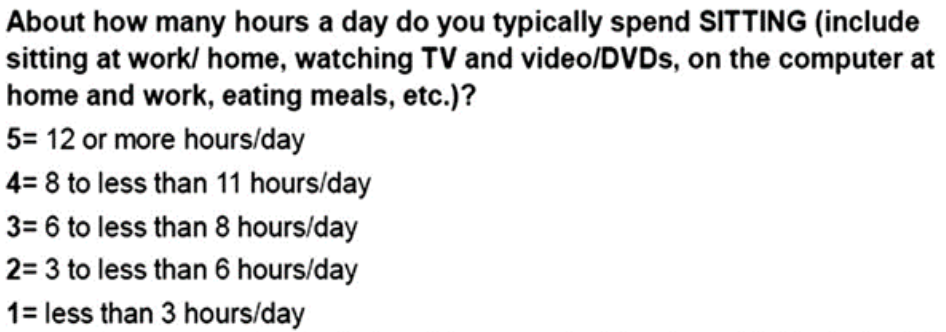 & $A$ & B & C \\
\hline Total of each column & & & \\
\hline
\end{tabular}

Cumulative INACTIVITY RISK INDEX (columns $a+b+c$ ): 